\title{
Práticas de gestão de mudança organizacional: Impacto nas atitudes e nos resultados percebidos com a mudança
}

\author{
Lílian Cristina Palhares Machado e Elaine Rabelo Neiva \\ Universidade de Brasília
}

\section{Resumo}

As práticas de gestão de mudança são intervenções organizacionais que facilitam a promulgação de processos de mudança nas empresas. O presente estudo testa a relação entre o uso das práticas de gestão da mudança e seus impactos sobre a atitude e sobre os resultados percebidos com a mudança. A amostra contou com 214 participantes de organizações brasileiras $(58,5 \%$ mulheres e $40,5 \%$ homens $)$. Os instrumentos utilizados foram a escala de percepção de mudanças organizacionais, avaliando os resultados transformacionais e transacionais percebidos; a escala de atitudes em relação a mudanças - avaliando ceticismo, temor e aceitação das mudanças e o questionário de avaliação das práticas de gestão de mudança organizacional, para avaliar cinco práticas de mudança organizacional (diagnóstico e alinhamento, liderança, comunicação, compensação e incentivos e treinamento). Como resultado verificou-se que as práticas de gestão de mudança impactam parcialmente as atitudes e os resultados percebidos com a mudança.

Palavras-chave: práticas de gestão de mudança, percepção, atitude 


\title{
Change management practices: Impact on attitudes and perceived results with change
}

\begin{abstract}
Change management practices are organizational interventions that facilitate the enactment of change processes in companies. This study tests the relationship between the use of change management practices and their impacts on attitude and on the results perceived by the change. The sample had 214 participants from Brazilian organizations (58.5\% women and $40.5 \%$ men). The instruments used were the change organizational perception scale, assessing transformational and transactional perceived results; the attitudes of scale in relation to changes - assessing opposition, fear and acceptance of change and the Questionnaire Assessment of Organizational Change Management Practices to evaluate five organizational change practices (diagnosis and alignment, leadership, communication, compensation and incentives and training). As a result, it was found that change management practices affect attitudes and results perceived by the change partly.
\end{abstract}

Keywords: change management practices, perception, attitude 


\section{Introdução}

Neiva e Paz (2012) abordam a mudança organizacional como alterações nos componentes organizacionais desencadeadas intencionalmente ou não por intervenções que tragam consequências para os resultados da organização. As autoras ressaltam ainda a necessidade de monitoramento e avaliação desse processo para evitar consequências danosas para os membros da organização.

Burnes (2004) descreve a mudança como algo presente na vida organizacional tanto no nível operacional quando estratégico. Burtonshaw-Gunn e Salameh (2010) afirmam que as mudanças se tornaram um componente diário das organizações como parte do desejo contínuo de melhoria no desempenho e da necessidade de aumentar o desempenho da organização e tornar esse empreendimento um processo bem sucedido gerou a necessidade de gestão da mudança.

O acompanhamento e o gerenciamento das emoções e reações humanas com o objetivo de reduzir uma queda inevitável de produtividade que é consequência dos processos de mudança, tem sido apontado por alguns autores como o objetivo maior da gestão da mudança organizacional (Maurer, 2001; Rao, 2015). Assim, fomentar atitudes positivas em relação ao processo e evitar a resistência a mudança parece ser o grande propósito da gestão da mudança, o que implica que a gestão da mudança é uma habilidade gerencial bastante necessária (Hayes, 2007; Senior, 2002).

Outros autores enfatizam o alcance dos resultados desejados como o grande propósito da gestão da mudança. $\mathrm{O}$ uso deliberado e consciente de estratégias, métodos sistemáticos e conhecimentos para garantir que a organização mude na direção dos resultados desejados e para garantir sua efetividade são características essenciais da gestão da mudança para vários autores (Davis \& Holland, 2002; Hayes, 2007).

Assim, concebendo a gestão da mudança em termos de práticas (Jansson, 2013; Rao, 
2015; Sande, Walela \& Wamukoya, 2015; Sims, 2002), este estudo avalia em que medida tais práticas influenciam as atitudes dos empregados frente a mudança e os resultados percebidos com o processo de mudanças. Considerando ainda que Damanpour (1991), Lines (2005) e Neiva e Paz (2012) afirmam que os resultados alcançados com a mudança e o sucesso das mudanças são afetados pelas atitudes dos indivíduos frente a mudança, este estudo postula um efeito mediador das atitudes na relação entre as práticas de gestão e os resultados percebidos com a mudança.

\section{Gestão da mudança organizacional}

O termo mudança organizacional envolve uma série de intervenções, ações humanas, atividades de comunicação e interação que não pode ser visto como estático. Assim, a mudança organizacional pode ser caracterizado como um processo (Jian, 2011; Schreyogg \& Sydow, 2011).

A mudança organizacional é um processo complexo, em que o motor da mudança desenvolve e é desenvolvido no decorrer do processo, e onde o velho e o novo se entrelaçam, cumulativamente, na construção de uma dinâmica inovadora (Castel \& Friedberg, 2009). Processo de mudança organizacional não é necessariamente linear, porque pode evoluir em ambos os sentidos, progressista e retrógrado (Ambos \& Birkinshaw, 2010), em retrocesso, numa espécie de redesenvolvimento, para o estado original antes que a regressão ocorreu. Assim, a mudança não acontece por acaso, ela é interativa por natureza (Castel \& Friedberg, 2009), mas a literatura sempre levanta a possibilidade de controle e monitoramento por parte dos gestores (Balogun, 2006; Balogun \& Hailey, 2004; Burke, 2011; Cinite, Duxbury, \& Higgins, 2009). Assim o termo gestão da mudança se remete a esta possibilidade de planejamento, controle e monitoramento da ação humana e das reações dos empregados no processo de mudança (Hayes, 2007; Maurer, 2001; Senior, 2002; Rao, 2015). 
O termo gestão da mudança tem sido utilizado com perspectivas diferentes na literatura. Para alguns autores, o termo envolve o acompanhamento e o gerenciamento das emoções e reações humanas com o objetivo de reduzir uma queda inevitável de produtividade que é consequência dos processos de mudança (Maurer, 2001). Evitar a resistência a mudança parece ser o grande propósito da gestão da mudança; o que implica que a gestão da mudança é uma habilidade gerencial bastante necessária (Hayes, 2007; Senior, 2002). O uso deliberado e consciente de estratégias, métodos sistemáticos para garantir que a organização mude na direção dos resultados desejados e para garantir sua efetividade são características essenciais da gestão da mudança para outros autores (Davis \& Holland, 2002; Hayes, 2007).

Sellmann e Marcondes (2010) definem gestão da mudança como “um recurso estratégico de organizações que auxilia no gerenciamento e execução de diversas fases do processo de mudança, que atuam com elevada complexidade e restrições em sua estrutura, sendo assim, um meio importante para manutenção da competitividade organizacional” (p. 753). Assim, a gestão da mudança se concentra na compreensão e execução do processo de mudança, ou seja, em definir "como" será realizada a mudança (processo) e em identificar "o que" precisa ser mudado (conteúdo). É importante ressaltar que todo o processo de mudança aspectos interpessoais (pessoas), tais como, a motivação, a comunicação, entre outras (Sellman \& Marcondes, 2010).

A tabela 1 apresenta algumas fases importantes para qualquer tipo de processo de mudança de acordo com Selmann e Marcondes (2010), fazendo menção aos autores que citaram as etapas em literatura. 
Tabela 1

Fases do processo de mudança

\begin{tabular}{l|l}
\hline \multicolumn{1}{c|}{ Fases } & \multicolumn{1}{c}{ Referências } \\
\hline $\begin{array}{l}\text { 1. Criação do senso de urgência pertinente. } \\
\begin{array}{l}\text { 2. Estabelecimento de um estado, através da evidência da } \\
\text { crise, problema ou oportunidade, no qual a complacência } \\
\text { deverá estar praticamente ausente. }\end{array}\end{array}$ & $\begin{array}{l}\text { 2000, 2001; Hayes, 2002; } \\
\text { Burnes, 2004) }\end{array}$ \\
\hline $\begin{array}{l}\text { 3. Estabelecimento de uma coalizão administrativa: } \\
\text { formação de um grupo com autoridade suficiente para } \\
\text { liderar a mudança. }\end{array}$ & (Kotter, 2000; Hayes, 2002) \\
\hline $\begin{array}{l}\text { 4. Alinhamento com a visão: estabelecimento de } \\
\text { consistência entre o processo de mudança e a visão da } \\
\text { empresa. }\end{array}$ & $\begin{array}{l}\text { (Adizes, 1995) (Kirkbridge, } \\
\text { 1998; Quatro, Hoekstra \& } \\
\text { Gilley, 2002) }\end{array}$ \\
\hline $\begin{array}{l}\text { 5. Capacitação de pessoas para atuarem conforme a visão: } \\
\text { construção do empowerment do grupo através da } \\
\text { eliminação de obstáculos ao processo de transformação e } \\
\text { encorajamento para correr riscos. }\end{array}$ & $\begin{array}{l}\text { (Adizes, 1995) (Kirkbridge, } \\
\text { Gilley, 2002) }\end{array}$ \\
\hline $\begin{array}{l}\text { 6. Planejamento e promoção de conquistas de curto } \\
\text { prazo: estabelecimento de metas a curto prazo para serem } \\
\text { atingidas e comemoradas }\end{array}$ & $\begin{array}{l}\text { (Anderson \& Anderson, 2001; } \\
\text { Quatro, Hoekstra, \& Gilley, } \\
\text { 2002) }\end{array}$ \\
\hline
\end{tabular}

Todd (1999) assume que a gestão da mudança é uma abordagem estruturada e sistemática para alcançar mudança sustentável no comportamento humano dentro da organização com foco no processo e nas pessoas. Stoner, Freeman e Gilbert (2009) afirmam que as rotinas e experiências organizacionais inevitavelmente agrupam precedentes que se tornam difíceis de mudar. Não se trata de aplicar regras duras e rápidas, mas direcionar um processo imprevisível, dependente do contexto, em que as estratégias podem levar a ambos os resultados intencionais e não intencionais. Mudança não é apenas dinâmica, emergente e não-linear, mas também frustrante e desanimadora, uma vez que, cerca de 70 por cento de todos os programas de mudança falham (Balogun, 2006; Balogun \& Hailey, 2004; Burke, 2011; Cinite et al., 2009). Pode-se sugerir que esta fraca taxa de êxito dos processos de mudança (Balogun, 2006; Balogun \& Hailey, 2004; Burke, 2011; Cinite et al., 2009) indica a falta de um quadro teórico válido de como implementar e gerenciar a mudança, pois o que 
está atualmente disponível para acadêmicos e profissionais é uma grande gama de teorias e abordagens (Burnes, 2004) contraditórias e confusas.

Os autores concordam sobre alguns temas que envolvem o tópico gestão da mudança (Burnes, 2004). A investigação sobre mudança organizacional diz essencialmente respeito aos temas como os processos de mudança (Schreyogg e Sydow, 2011), liderança (Battilana, Gilmartin, Sengul, Pache, \& Alexander, 2010), execução de mudança (Van de Ven \& Sun, 2011), recepção da mudança dentro da organização, as reações dos empregados (Oreg, Vakola, \& Armenakis, 2011), o discurso (Grant \& Marshak, 2011), ou paradoxos (Smith \& Lewis, 2011).

Mesmo que não haja uma ampla aceitação de uma única abordagem clara e prática para a organização sobre gerenciamento de mudanças que explique o que muda as organizações, o que as organizações precisam fazer e como implementar as mudanças (Burnes, 2004), a abordagem planejada para a mudança organizacional apresenta tentativas de explicar o processo que gera mudanças (Eldrod II \& Tippett, 2002). Além disso, a abordagem planejada enfatiza a importância de compreender os diferentes estados que uma organização terá para percorrer, a fim de passar de uma situação insatisfatória à identificação do estado desejado (Eldrod II \& Tippett, 2002).

A mudança organizacional planejada significa aumentar os níveis de estresse do empregado intencionalmente (Dahl, 2011), perturbar as operações em curso com conhecimento de causa (Currie et al, 2009;. Pache \& Santos, 2010) e conscientemente jogar com o futuro da organização, pois o resultado é muitas vezes diferente do plano original (Jian, 2011;. Whittle, Suhomlinova, \& Mueller, 2010). Assim, as práticas de mudança organizacional são do interesse de ambos - os acadêmicos e profissionais.

Vários autores ressaltam que a gestão da mudança organizacional deve ser compreendida em termos de práticas (Jansson, 2013; Sims, 2002). Segundo Sande, Walela \& 
Wamukoya (2015), os processos de mudança bem-sucedidos possuem foco nas melhores práticas de gestão. Rao (2015), a partir de um estudo empírico, revelam que a aceitação do que é proposto pelas pessoas, os sistemas de incentivo, o foco em tecnologia, a aprendizagem, formação de equipes e líderes, o compromisso com os objetivos organizacionais, visão e estratégia, a comunicação compartilhada das mudanças geram vitórias de curto prazo. Os autores ressaltam também que as práticas de gestão da mudança são ferramentas para organizações eficazes e competitivas, porque conectam as crenças das pessoas fornecendo uma ideia clara sobre onde estar na próxima fase.

\section{Práticas de gestão da mudança organizacional}

O termo prática possui muitas definições. Práticas podem ser "arranjos materialmente encarnados e mediados da atividade humana, centralmente organizados em torno entendimento prático e compartilahdo"(Schatzki, 2001, p. 2), ou "formas conectadas, compartilhadas e implícitas de compreender, dizer, e fazer as coisas" (Whittle, Suhomlinova, \& Mueller, 2009, p. 31).

Muito pouco consenso existe sobre quais práticas podem ser específicas da gestão da mudança (Jansson, 2013). Comentários sobre tópicos de gestão da mudança organizacional existem, mas a sua análise é apresentada numa perspectiva específica, como reações à mudança (Oreg, Vakola, \& Armenakis, 2011;. Hardy \& Thomas, 2011)., características de dualidade na organização ou sustentação da mudança organizacional como a mudança se desdobra, como mudança e continuidade são equilibradas em conjuntos de simultânea processos (Sonenshein, 2010).

Embora a relevância de conteúdo e do contexto de mudança organizacional seja reconhecida entre os estudiosos, uma suposição dominante parece ser de que práticas de mudança organizacional são de natureza universal (Jansson, 2013; Kotter, 2007; 
Martin, Johnson, \& Cullen,, 2009; Schaffer, 2010). Além desse pressuposto, Jansson (2013) afirma que existem dois outros pressupostos. O segundo pressuposto de investigação das práticas de gestão da mudança envolve a resistência encarada como resultado da mudança (Danisman, 2010), constituindo um fenómeno natural e certo decorrente da (Levay, 2010) mudança. Por exemplo, uma vasta revisão de 60 anos de estudos quantitativos sobre as reações de dos alvos da mudança organizacional, por Oreg et al. (2011), mostra que estudos enfocando razões para resistir à mudança e como afetar essa resistência são marginais.

A terceira suposição indicada por Jansson (2013) consiste na ideia de que os práticos, ou consultores agem como membros de grupos definidos pela hierarquia organizacional. Estes supostos grupos profissionais são, por exemplo a gestão de topo (Barron, Riby, Greer, \& Smallwood, 2011; Zhang \& Rajagopalan, 2010), a média gerência (Luscher \& Lewis, 2008;. Rouleau Balogun, 2011) ou os agentes de mudança (Schwarz et al, 2011;. Stensaker \& Langley, 2010).

De acordo com Jansson (2013) as práticas são dimensões de atividade dentro de contexto socialmente construído, como regras, ferramentas, métodos, reuniões, práticas sociais e práticas discursivas (Jarzabkowski \& Seidl, 2008). Na verdade, a aplicação de práticas universais como "melhores práticas" pode condicionar mudança organizacional ao emprego de regras na prática, por meio de técnicas menos contextualizadas, superenfatizando fatores e ignorando as normas discursivas.

A despeito da popularidade das práticas de gestão de mudança organizacional, surge a pergunta se as suas receitas são baseadas em evidências sólidas e convergentes. Para responder a essa pergunta, Barends, Janssen, ten Have e ten Have (2014) realizaram uma revisão sistemática sobre gestão de mudança organizacional com um total de 563 estudos. A avaliação dos autores mostra um predominância de estudos one-shot com uma validade interna baixa. Estudos de replicação são raros. Os resultados sugerem que os estudiosos e 
profissionais devem ser céticos em relação ao corpo dos resultados da investigação no domínio da gestão de mudança organizacional publicados.

Mesmo havendo descrença, Woodman (2014) afirma que estudiosos oferecem interessantes insights para práticas de mudança organizacional, tais como abordagens de liderança, métodos de planejamento, funcionamento de processos, estratégia, engajamento pessoal, gestão de conflitos e práticas de comunicação (Currie \& Lockett, 2009;. Ford, Ford, \& D'Amelio, 2008; Pache \& Santos, 2010; Stensaker et al., 2008). Sande, Walela, \& Wamukoya (2015) identificaram que os gestores escolares praticavam moderadamente aspectos de planejamento, liderança comprometida, alinhamento da força de trabalho, participação dos interessados e definição de estruturas de governança em suas instituições, descritos como práticas de gestão da mudança. O estudo descobriu que as práticas de gerenciamento da mudança adotadas pelas instituições influenciaram significativamente o desempenho dos indivíduos e das escolas como um todo.

Ranieri (2011) descreve as práticas de gestão de mudança como um "conjunto de práticas de intervenções organizacionais que quando executadas de forma adequada e em coerência com os eventos organizacionais internos e externos, facilitam a promulgação de processos de mudança organizacional” (p.226). O autor encontrou em seu estudo as práticas influenciando os resultados dos processos de mudança percebidos pelos indivíduos, embora não tenha utilizado um instrumento de pesquisa exclusivo para avaliar a percepção.

Ainda que haja abundância de literatura com conselhos sobre mudança de gestão para os profissionais (Ranieri, 2011), quatro práticas (diagnóstico e alinhamento organizacional, comunicação, liderança e compensação/ incentivo) são consideradas como frequentes nos estudos sobre gestão da mudança (Raineri, 2011). O mesmo autor sugeriu, nas considerações finais da sua pesquisa, a inserção de mais uma prática que é o treinamento.

A primeira prática é a identificação da necessidade ou a preparação para a mudança, 
também denominada de diagnóstico e análise (Buchanan, et al., 2005; Kotter, 2000). A segunda prática, liderança, aborda a compreensão e atuação do líder na necessidade de transformar, para que seus liderados também se comprometam com a mudança (Kotter, 2000; Battilana et al., 2010).

A comunicação é a terceira prática porque permite entender o que, quando e por que a organização está mudando, o que facilita a aceitação e adaptação das novas circunstâncias desde o nível estratégico até o operacional (Lewis, Schmisseur, Stephens, \& Weir, 2006; Kotter, 2000). As duas últimas práticas de gestão de mudança são compensação e incentivos (Afande, 2015; Kotter, 2000) e o treinamento que prepara os empregados para a mudança (Sellmann \& Marcondes, 2010; Pierce \& Sims, 2002).

\section{Percepção dos resultados da mudança}

A percepção dos resultados da mudança é a maneira de observar as alterações realizadas por um processo de mudança, considerando critérios usados nas tipologias de mudança. (Domingos \& Neiva, 2014; Neiva \& Paz, 2012;).

Weick e Quinn (1999) constatam que existem dois grandes tipos de mudanças que são analisados pela literatura: as mudanças Contínuas, que envolvem pequenos avanços ao longo do tempo, sendo cumulativas, e as mudanças Episódicas; resultado do desequilíbrio organizacional. Segundo Van de Ven e Poole (2005) essa diferenciação reflete tendências do estudo e visões de organizações bem como abordagens metodológicas diferenciadas no estudo da mudança organizacional.

Burke (2011a); Burke (2011b) e Burke e Litwin (1992) propõem uma diferenciação entre mudança Transformacional e mudança Transacional. Segundo os autores, a mudança Transformacional possui grande impacto, consequência da interação e das pressões do ambiente externo, demandando novos comportamentos dos empregados e alterando toda a 
organização. Envolve a ruptura de padrões anteriores, atingindo a organização em sua totalidade. Exige uma reconfiguração e redirecionamento; a criação de novas estratégias, atividades, missão, a organização dos processos, de estrutura e de pessoas. Segundo os autores, o outro tipo de mudança - a mudança Transacional - é gerado pela atuação interna da organização sobre fatores individuais, grupais e organizacionais que estão relacionados às operações (transações) do dia-a-dia dos indivíduos no ambiente organizacional. Ela altera apenas alguns aspectos da organização e, pressupondo a estabilidade externa, faz pequenos ajustes continuamente visando à eficiência organizacional. Ela não exige alteração da identidade, dos valores ou da missão. Os tipos de mudanças Transformacionais e Transacionais apresentadas por Burke e Litwin (1992) correspondem, respectivamente, aos tipos de mudança Episódica e Contínua relatados por Weick e Quinn (1999).

A tipologia de Mudanças Transformacionais e Transacionais proposta por Burke e Litwin (1992) foi utilizada neste trabalho como origem da análise das mudanças ocorridas na organização pesquisada, considerando os resultados efetivos, pois estabelece conteúdos que possam ser usados para os avaliar os resultados da mudança.

A primeira contribuição deste trabalho é testar o impacto que a utilização práticas de gestão da mudança tem nos resultados da mudança organizacional. Como foi dito anteriormente, a maioria da literatura afirma que o uso dessas práticas tem um efeito positivo nos resultados e objetivos a serem alcançados com as mudanças organizacionais. No entanto, muito pouca evidência empírica existe para apoiar este argumento. Este artigo testa a relação entre as percepções sobre o uso das prática de gestão da mudança, a realização dos objetivos do programa de mudança e o seu impacto sobre os resultados percebidos.

Os autores enfatizam o alcance dos resultados desejados como o grande propósito da gestão da mudança. O uso deliberado e consciente de estratégias, métodos sistemáticos e conhecimentos para garantir que a organização mude na direção dos resultados desejados e 
para garantir sua efetividade são características essenciais da gestão da mudança para vários autores (Davis \& Fisher, 2002; Hayes, 2007). Assim, apresenta-se a hipótese 1 deste estudo: Hipótese 1: As práticas de gestão da mudança afetam os resultados percebidos pelos indivíduos com o programa de mudanças.

\section{Atitudes frente a mudança organizacional}

Nastase, Giucleia e Bold (2012) afirmam que o fomento de atitudes positivas dos indivíduos frente a mudança organizacional é objetivo específico das práticas de gestão da mudança. Neiva e Pantoja (2011) afirmam que as atitudes dos indivíduos frente à mudança desempenham um papel fundamental na relação entre indivíduos e mudança organizacional. As atitudes dos indivíduos frente à mudança organizacional conquistam a importante característica de facilitadores de mudança, pois asseguram sucesso à notoriedade dos processos de mudança organizacional.

Lines (2005) apresenta uma nova proposta conceitual para analisar as atitudes e suas consequências comportamentais. De acordo com o autor, as atitudes seriam classificadas em fortes e fracas, positivas e negativas e comportamentos específicos surgiriam a partir da interrelação entre essas dimensões. A resistência, por exemplo, seria resultado de atitudes negativas e fortes em relação à mudança, esclarece Neiva e Pantoja (2011).

Bortolotti, Junior e Andrade (2009) afirmam que: "do ponto de vista individual, a mudança pode originar emoções e reações que variam do otimismo ao medo, podendo incluir ansiedade, resistência, desafio, energia, ambiguidade, entusiasmo, incapacidade, receio, motivação e pessimismo" (p.2), bem como crenças, atitudes e intenções comportamentais dos empregados (Self, Armenarkis, \& Schraeder, 2007).

Assim, concebendo a gestão da mudança em termos de práticas (Jansson, 2013; Rao, 2015; Sande, Walela \& Wamukoya, 2015; Sims, 2002), a literatura aponta que o 
acompanhamento e o gerenciamento das emoções e reações humanas com o objetivo de reduzir uma queda inevitável de produtividade que é consequência dos processos de mudança tem sido observado por alguns autores como o objetivo maior da gestão da mudança organizacional (Maurer, 2001; Rao, 2015). Assim, fomentar atitudes positivas em relação ao processo e evitar a resistência a mudança parece ser o grande propósito da gestão da mudança, o que implica que a gestão da mudança é uma habilidade gerencial bastante necessária (Hayes, 2007; Senior, 2002). A partir daí surgem as hipóteses 2 e 3 deste estudo:

Hipótese 2: As práticas de gestão da mudança afetam positivamente as atitudes de aceitação da mudança organizacional;

Hipótese 3: As práticas de gestão da mudança afetam negativamente as atitudes de ceticismo e de temor frente à mudança organizacional.

De maneira geral, literatura assinala que acompanhamento e o gerenciamento das emoções e reações humanas possuem o objetivo final de reduzir uma queda inevitável de produtividade que é consequência dos processos de mudança e promover o alcance dos objetivos e resultados pretendidos com a mudança (Maurer, 2001; Rao, 2015), o que pode configurar a mediação das atitudes frente a mudança organizacional na relação entre as práticas de gestão da mudança e os resultados percebidos com a mudança organizacional. Algumas prescrições (Lines, 2005) e algum suporte empírico (Damanpour, 1991; Neiva \& Paz; 2012) sustentam que os resultados alcançados com a mudança e o sucesso das mudanças são afetados pelas atitudes dos indivíduos frente a mudança. As hipóteses 4 e 5 são apresentadas:

Hipótese 4: As atitudes de aceitação frente a mudança organizacional medeiam a relação entre as práticas de gestão da mudança organizacional e os resultados percebidos com a mudança; 
Hipótese 5: As atitudes de ceticismo e temor frente a mudança organizacional medeiam a relação entre as práticas de gestão da mudança organizacional e os resultados percebidos com a mudança.

\section{Método}

Os participantes desta pesquisa foram pessoas de diferentes organizações que já tivessem passado por processos de mudança nos últimos 12 meses. A amostra foi por acessibilidade. A pesquisa possui o caráter de recorte transversal, com amostra inicial de 214 sujeitos. A aplicação dos instrumentos foi realizada por meio de um site da Internet, onde um link para acesso dos instrumentos foi divulgado em redes sociais, em que o indivíduo respondia às questões e estas eram enviadas e armazenadas em um banco de dados. Os instrumentos ficaram disponíveis aos interessados em participar pelo período de trinta dias.

Além das escalas utilizadas, o instrumento contou com os dados demográficos: idade, sexo, grau de escolaridade, tipo e ramo de negócio da empresa em que trabalha, tempo de empresa, região em que trabalha e se o participante exercia ou não cargo de liderança.

\section{Instrumentos}

Foram utilizadas, como instrumentos de pesquisa, três escalas validadas psicometricamente, aplicadas por meio eletrônico.

A Escala de Percepção de Mudanças Organizacionais (EPMO) - 13 itens com indícios de validade apontados por Domingos e Neiva (2014) e Picchi (2010). Esta escala avalia os resultados dos tipos de mudança transacional (ou contínua) e transformacional (ou radical). A Escala de Atitude Frente às Mudanças (27 itens) com indício de validade apresentados por Neiva, Ros e Paz (2004), que avalia as atitudes de ceticismo, temor e aceitação das mudanças organizacionais. E para avaliar as cinco práticas de mudança 
organizacional (diagnóstico e alinhamento, liderança, comunicação, compensação e incentivos e treinamento) foi utilizado o questionário de Avaliação das Práticas de Gestão de Mudança Organizacional (20 itens) para os receptores da mudança com indícios de validade encontrados por Ranieri (2011). Para os três instrumentos foram utilizadas escala de concordância tipo Likert de 10 pontos (1 para Discordo Plenamente e 10 para Concordo Plenamente).

\section{Participantes}

Responderam a pesquisa 214 sujeitos, sendo 58,5\% mulheres $(\mathrm{N}=120)$ e 40,5\% homens $(\mathrm{N}=83) .20 \%$ dos respondentes têm entre 19 e 25 anos $(\mathrm{N}=41), 42,9 \%$ entre 26 e 35 anos $(\mathrm{N}=88), 22,4 \%$ entre 36 e 45 anos, $11,2 \%$ entre 46 e 55 anos $(\mathrm{N}=23)$ e $3,4 \%$ acima de 56 anos $(\mathrm{N}=7)$. Sobre a escolaridade 2,9\% possuem nível médio $(\mathrm{N}=6), 36,1 \%$ nível superior incompleto $(\mathrm{N}=74), 37,6 \%$ nível superior completo $(\mathrm{N}=77), 16,6 \%$ especialização/MBA $(\mathrm{N}=34), 5,9 \%$ possuem mestrado $(\mathrm{N}=12)$ e $1 \%$ doutorado $(\mathrm{N}=2)$. Quanto ao tipo de empresa dos sujeitos pesquisados, $73,7 \%$ trabalham em empresas privadas $(\mathrm{N}=151), 18,5 \%$ empresas públicas $(\mathrm{N}=38), 5,9 \%$ empresas de economia mista $(\mathrm{N}=10), 2 \%$ empresas sem fins lucrativos $(\mathrm{N}=4)$ e $1 \%$ não respondeu $(\mathrm{N}=2)$. Com relação ao ramo das empresas, $36,6 \%$ dos sujeitos trabalham em empresas na área de prestação de serviço $(\mathrm{N}=75), 22,9 \%$ no ramo comercial $(\mathrm{N}=47), 15,6 \%$ empresas governamentais $(\mathrm{N}=32), 8,3 \%$ no ramo educacional, $6,3 \%$ na construção civil $(\mathrm{N}=13), 4,9 \%$ no ramo da indústria $(\mathrm{N}=10), 3,4 \%$ na saúde $(\mathrm{N}=7)$, $1 \%$ no agronegócio $(\mathrm{N}=2)$ e $1 \%$ não respondeu $(\mathrm{N}=2)$. Quanto ao tempo de empresa, $27,3 \%$ dos sujeitos tem até 1 ano de empresa $(\mathrm{N}=56), 16,1 \%$ possui entre 1 e 2 anos de empresa $(\mathrm{N}=33), 23,4 \%$ entre 2 e 4 anos $(\mathrm{N}=48), 12,7 \%$ entre 4 e 6 anos $(\mathrm{N}=26), 19,5 \%$ acima de 6 anos $(\mathrm{N}=40)$ e $1 \%$ não respondeu $(\mathrm{N}=2)$. Quanto à função exercida, 63,9\% das respostas são de funcionários que não exercem cargo de liderança $(\mathrm{N}=131), 33,7 \%$ exercem cargos de 
liderança $(\mathrm{N}=69)$ e 2,4\% não respondeu $(\mathrm{N}=5)$. Com relação às regiões em que os sujeitos trabalham, $92,7 \%$ trabalham no centro-oeste $(\mathrm{N}=190), 3,4$ na região sudoeste $(\mathrm{N}=7), 1,5 \%$ no sul $(\mathrm{N}=3)$, e $1 \%$ no nordeste $(\mathrm{N}=2), 1,5 \%$ não respondeu $(\mathrm{N}=3)$.

\section{Análise de Dados}

Foram realizadas as análises fatoriais dos instrumentos por meio do software estatístico Statistical Package for Social Science - SPSS, versão 20.0.

Inicialmente foram analisados os pressupostos das análises estatísticas multivariadas, são elas: normalidade, linearidade, homocedasticidade, multicolinearidade, singularidade, dados atípicos e ausentes. Para realização das análises estatísticas foram consideradas apenas as variáveis que tinham menos de $10 \%$ dos seus itens em branco (Field, 2013). Na sequência foi realizada análise exploratória para tratamento dos dados atípicos, utilizando a média junto com as medidas de dispersão (desvio padrão, coeficiente de variância e erro amostral).

Nas análises fatoriais, foram avaliadas as fatorabilidades das matrizes pelas medidas de adequação da amostra Kaiser-Meyer-Olkin (KMO), depois a Análise dos Componentes Principais (PCA), sendo que para estimar o número de fatores do instrumento utilizou-se dos critérios de: eigenvalue superior a 2,0, gráfico ScreePlot, porcentagem de variância explicada e análise paralela. Para a extração dos fatores, utilizou-se a Análise Fatorial dos Eixos Principais (PAF) e o método de rotação Promax. O coeficiente Alfa de Cronbach maior que 0,70 foi utilizado para medir a confiabilidade de cada fator.

\section{Resultados}

\section{Análise Fatorial}

Para confirmação das estruturas fatoriais encontradas em amostras anteriores, foram realizadas novas análises fatoriais dos instrumentos de Resultados Percebidos (Picchi, 2010; 
Domingos \& Neiva, 2014) e Atitude Frente à Mudança (Neiva, Ros \& Paz, 2004). No caso do instrumento de Práticas de Gestão da Mudança, as análises fatoriais foram realizadas com objetivo de verificar se a estrutura fatorial obtida por Raineri (2011) se mantinha em uma amostra brasileira.

\section{Resultados referentes à Análise Fatorial de Resultados Percebidos}

Após o tratamento e limpeza dos dados, foi obtida uma amostra de 210 sujeitos. A razão de casos por variável foi de 16,15 sujeitos por item. A análise do eigenvalue superior a 1 e dos componentes principais indicaram 3 fatores com variância explicada maior que $3 \%$. Porém, pelos critérios do gráfico ScreePlot e a Análise Paralela, a solução indicada foi a de 2 fatores, aqui adotada. De acordo com os resultados obtidos, a variância total explicada pelo instrumento composto por 13 itens distribuídos em 2 fatores foi de 39,40\%. Os dois fatores encontrados foram denominados Transformacional e Transacional, confirmando a estrutura anteriormente encontrada. Foi analisada a coerência dos itens que compunham cada fator e excluídos os itens de número 01, 02, 04, 07 e 09 por possuírem carga fatorial inferior a 0,45 (Tabachnick \& Fidel, 2012). Foram mantidos os 4 itens de RP - Transformacional apresentaram cargas fatoriais variando entre 0,467 e 0,790 e Alfa de Cronbach de 0,70. Os 4 itens relacionados a RP - Transacional apresentaram cargas fatoriais entre 0,513 e 0,704 e Alfa de Cronbach de 0,70. Esses dados reproduzem a estrutura encontrada por estudos anteriores (Picchi, 2010; Domingos \& Neiva, 2014).

\section{Resultados referentes à Análise Fatorial das Atitudes Frente à Mudança}

Após o tratamento e limpeza dos dados, foi obtida uma amostra de 207 sujeitos. As análises iniciais do instrumento indicaram a presença de dados faltosos em 25 dos 31 itens da 
escala, a técnica de regressão foi utilizada para substituir os casos faltosos. Estes casos faltosos foram inferiores a $3 \%$ em todas as variáveis.

Adotando o critério de 5 a 10 casos por item (Pasquali, 2012), tem-se 6,6 casos por variável, sendo uma amostra robusta para avaliação de novos indícios de validade da escala.

Foi realizada então a fatorabilidade da matriz pelo indicador KMO, esta medida de adequação amostral teve um resultado de 0,84 , índice considerado meritório por Pasquali (2012), indicando uma boa adequação dos dados à análise fatorial.

Com base no gráfico ScreePlot, considerando os Eigenvalues acima de 2, a análise paralela e o percentual de variância explicada por cada fator, decidiu-se pela extração de 3 fatores pelo método de análise fatorial dos eixos principais (Principal Axis Factoring), rotação Promax.

Foram analisadas a carga fatorial de cada item, itens com carga menor que 0,45 (Tabachnick \& Fidel, 2012) foram excluídos, e a coerência dos itens que compunham cada fator, uma vez que o fator é considerado bom quando o conjunto de itens que o compõem possuem conceitos teóricos compatíveis ao que ele representa (Pasquali, 2012). Foram excluídos os itens 01, 07, 11 e 17.

A solução fatorial dos três fatores encontrados, explica 50,21\% da variância total, com os respectivos itens, as cargas fatoriais, percentual de variância explicada e Alfa de Cronbach. Portanto, o instrumento de Atitudes Frente à Mudança apresenta-se com 3 fatores, O Fator 1, denominado Aceitação, com 12 itens, com cargas fatoriais variando entre 0,46 e 0,74 e alfa de Cronbach de 0,89. O Fator 2, denominado Ceticismo, com 9 itens, cargas fatoriais variando entre 0,50 e 0,77 e alfa de Cronbach de 0,87. E, o Fator 3, Temor, com 6 itens, cargas fatoriais variando entre 0,45 e 0,83 e alfa de Cronbach de 0,85.

\section{Resultados referentes à Análise Fatorial de Práticas de Gestão da Mudança}


Após o tratamento e limpeza dos dados, foi obtida uma amostra de 203 sujeitos. A razão de casos por variável foi de 11,27 sujeitos por item. A medida do KMO foi de 0,933, enquadrando-se no nível "maravilhoso" (Pasquali, 2012).

A análise do eigenvalue superior a 1 e dos componentes principais indicaram 3 fatores com variância explicada maior que 3\%. Porém, pelos critérios do gráfico ScreePlot e a Análise Paralela, a solução indicada foi a de 1 fator, aqui adotada.

De acordo com os resultados obtidos, a variância total explicada pelo instrumento composto por 20 itens foi de 53,64\%. Os 20 itens de Práticas de Gestão da Mudança apresentaram cargas fatoriais variando entre 0,491 e 0,812 e Alfa de Cronbach de 0,954.

A Tabela 01 apresenta a solução fatorial do instrumento, com os respectivos itens, as cargas fatoriais, percentual de variância explicada e Alfa de Cronbach.

\section{Tabela 1}

Matriz Pattern de Práticas de Gestão da Mudança com o percentual de variância explicada e Alfa de Cronbach.

\begin{tabular}{ll}
\hline \hline & Fator 1 \\
\hline $\begin{array}{l}\text { 07 Os objetivos do programa de mudança foram estabelecidos com clareza para toda a } \\
\text { empresa. }\end{array}$ & 0,812 \\
$\begin{array}{l}\text { 15 Para facilitar o processo de mudança os funcionários receberam ajuda personalizada } \\
\text { quando necessitaram. }\end{array}$ & 0,806 \\
05 Foi dada, aos funcionários, a capacitação necessária para realizar os novos trabalhos. & 0,803 \\
$\begin{array}{l}\text { 17 A empresa se esforça para entender como seus funcionários compreenderam seus } \\
\text { comunicados. }\end{array}$ & 0,802 \\
$\begin{array}{l}\text { 10 Foram realizados treinamentos no local de trabalho para ajudar na adaptação à } \\
\text { mudança }\end{array}$ & 0,802 \\
$\begin{array}{l}\text { 11 Processos de trabalho foram analisados a fim de identificar as atividades e as áreas } \\
\text { que poderiam ser afetadas pelo programa de mudança. }\end{array}$ & 0,783 \\
$\begin{array}{l}\text { 19 Os funcionários receberam treinamentos no uso de novas habilidades. } \\
\text { 20 A empresa fez frequentes esforços de comunicação para garantir a compreensão e } \\
\text { apoio ao programa de mudança. }\end{array}$ & 0,780 \\
02 A empresa comunicou com clareza os objetivos e desafios individuais e setoriais. \\
$\begin{array}{l}\text { 08 Executivos demonstraram publicamente seu comprometimento com o processo de } \\
\text { mudança. }\end{array}$ & 0,776 \\
$\begin{array}{l}\text { 09 A empresa desenvolveu programas de incentivos com o objetivo de recompensar } \\
\text { comportamentos esperados. }\end{array}$ & 0,742 \\
$\begin{array}{l}\text { 13 Líderes fortemente carismáticos foram usados para dirigir o processo de mudança. } \\
\text { 14 A empresa identificou e recompensou o alcance parcial dos objetivos que estavam na } \\
\text { direção apropriada. }\end{array}$ & 0,731 \\
\end{tabular}


01 A empresa analisou seus pontos fortes e fracos para enfrentar o programa de mudança.

12 A empresa como um todo conhecia os planos de ação para promover o programa de mudança.

03 O programa de mudança foi liderado por indivíduos com alto grau de credibilidade dentro da empresa.

06 Oportunidades e ameaças existentes foram considerados durante o desenvolvimento do programa de mudança.

04 A empresa adotou programas de compensação com o objetivo de promover novas responsabilidades e demandas de trabalho.

18 A empresa premiou os seus funcionários quando esses atenderam os objetivos do programa de mudança.

Método de extração: Fatoração do Eixo principal.

a. 1 fatores extraídos. 3 iterações requeridas.

\section{Análise dos dados - Modelagem por Equações Estruturais (MEE)}

As análises estatísticas dos dados foram realizadas por meio do Statistical Package for Social Science - SPSS, versão 20.0 e do software AMOS 20.0.

Inicialmente foram analisados os pressupostos das análises estatísticas multivariadas, são elas: normalidade, linearidade, homocedasticidade, multicolinearidade, singularidade, dados atípicos e ausentes. Foram utilizadas variáveis com índice de dados ausentes inferiores a 10\% (Field, 2013). Todos os pressupostos estatísticos das análises foram confirmados de acordo com os critérios indicados por Field (2013).

A técnica de Modelagem por Equações Estruturais (MEE) foi utilizada para avaliar o modelo estrutural proposto, assim como para sugerir um modelo adequado de predição das práticas de gestão de mudança e resultados percebidos.

Para o teste de ajuste do modelo proposto foram analisados os seguintes índices: $\chi^{2}$, CFI (Comparative Fit Index); RMSEA (Root Mean Square Error of Aproximation); NFI (Normed Fit Index) e GFI (Goodness-of-fit Index), conforme recomendado pela literatura (Byrne, 2010; Kline, 2011). Adotaram-se, como critérios de ajuste satisfatório de modelo aos 
dados, os seguintes valores dos índices: CFI superior a 0,90; RMSEA próximo ou inferior a 0,12; GFI superior a 0,90 ; e NFI superior a 0,90 .

Os resultados das análises dos dados serão apresentados a seguir.

\section{Análises Descritivas}

Feito as análises fatoriais, foram realizadas as análises descritivas das variáveis: Resultados Percebidos, Atitudes Frente à Mudança e Práticas de Gestão da Mudança.

Tabela 2

Estatísticas Descritivas

\begin{tabular}{lcccccc}
\hline \hline & $\mathrm{N}$ & Mínimo & Máximo & Média & $\begin{array}{l}\text { Desvio } \\
\text { padrão }\end{array}$ & $\begin{array}{l}\text { Coeficiente } \\
\text { de Variação }\end{array}$ \\
\hline $\begin{array}{l}\text { Resultado transformacional } \\
\text { percebido }\end{array}$ & 205 & 2,14 & 9,50 & 5,76 & 1,75 & 0,30 \\
$\begin{array}{l}\text { Resultado transacional percebido } \\
\text { Atitudes de aceitação }\end{array}$ & 205 & 1,00 & 9,40 & 4,22 & 1,93 & 0,46 \\
Atitudes de ceticismo & 205 & 1,42 & 10,00 & 5,61 & 1,91 & 0,34 \\
$\begin{array}{l}\text { Atitudes de temor } \\
\begin{array}{l}\text { Pratica de gestão de mudança } \\
\text { (práticas) }\end{array}\end{array} \quad 205$ & 1,00 & 10,00 & 5,01 & 2,10 & 0,42 \\
\hline \hline
\end{tabular}

N válido (de lista): 205

Conforme observado na Tabela 02, os respondentes percebem um moderado efeito de mudança organizacional transformacional (M 5,76, DP=1,75), e um efeito muito pequeno de mudança organizacional transacional $(M=4,22, \mathrm{DP}=1,93)$, e também houve uma percepção muito pequena da utilização das Práticas de Gestão de Mudança (M=4,44, DP=2,04) fatores que se mantiveram próximo ao ponto médio da escala. Valores maiores são indicados pelos participantes quanto a Atitudes Frente à Mudança, nos fatores aceitação (M=5,61, DP=1,91), ceticismo (M=5,01, DP=2,10) e temor $(M=6,91, D P=2,13)$. 


\section{Resultados referentes ao Modelo Proposto}

Para avaliação do modelo proposto foi realizado Modelagem de Equação Estrutural (MEE). O modelo inicial apresentou um razoável ajuste, contudo ainda apresentada muito resíduo. Analisando os resultados e as sugestões do softaware, observou-se covariâncias significativas entre os erros, não previstas no modelo inicial. Essas covariâncias ocorrem entre os itens 1 e 3, 2 e 3 e 2 e 6 . De acordo com Kline (2011), até três covariâncias entre erros podem ser aceitas no modelo.

A Figura 01 apresenta o novo modelo testado e ajustado, já com os coeficientes estimados pela equação estrutural.

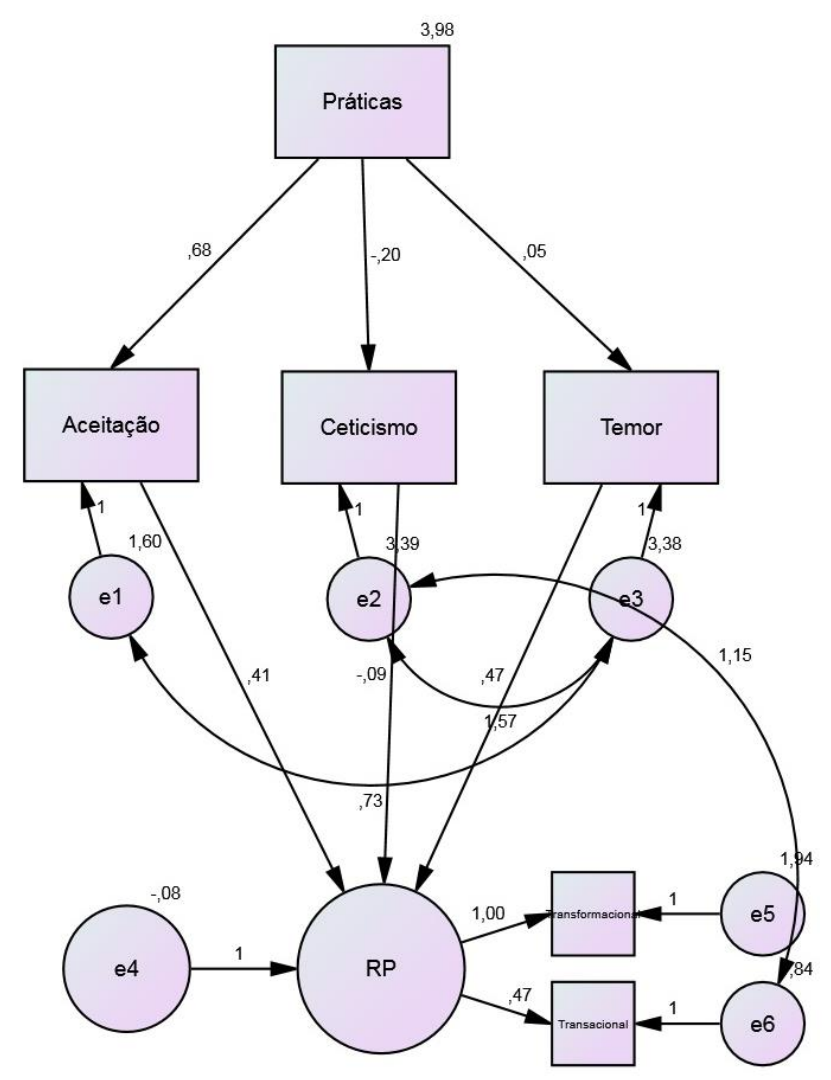

Figura 1. Modelo ajustado e resultados da equação estrutural 
Os índices de adequação do modelo inicial foram próximos ao satisfatório, como pode ser observado na Tabela 02. Porém, nem todos os parâmetros estimados no modelo testado são estatisticamente significativos.

A Tabela 03 apresenta os índices de adequação do novo modelo testado (na coluna modelo ajustado). Observa-se nessa tabela que houve uma melhora sensível nos índices de ajuste do modelo, após a adoção da correlação entre erros, em relação aos dados obtidos no primeiro modelo testado. A diferença dos valores do $\chi^{2}$ foi de 139,42 pontos que são significativos (dado GL=3). Além disso, todos os outros índices melhoraram. O RMSEA continua acima de 0,10 , isto sugere que o modelo ainda possui resíduo, o que indica que podem ser selecionadas outras variáveis para compor o modelo. Essa modificação ocorreu devido à real existência da correlação entre os erros dos itens (motivo do ajuste do modelo), e que não foi previsto inicialmente. A correlação em questão possui uma justificativa teórica, uma vez que os erros dos fatores que possuem covariância são de dois instrumentos baseados na mesma teoria de mudança organizacional. Sendo assim, o ajuste do modelo respeitou as recomendações de justificativa teórica para sua adoção (McDonald \& Ho, 2002).

Tabela 3

Índices de adequação do modelo

\begin{tabular}{lll}
\hline Índice & Modelo Inicial & Modelo Ajustado \\
\hline$\chi^{2}$ & $152,85(\mathrm{GL}=7)$ & $13,43(\mathrm{GL}=4)$ \\
GFI & 0,82 & 0,97 \\
CFI & 0,69 & 0,97 \\
NFI & 0,68 & 0,97 \\
RMSEA & 0,33 & 0,11 \\
\hline \hline
\end{tabular}

$\mathbf{\Delta}($ diferença $) \chi^{2}=139,42(\mathrm{GL}=3)$

GFI - Goodness-of-Fit Index; CFI - Comparative Fit Index; 
NFI - Normed Fit Index; RMSEA - Root Mean Square Error of

Approximation

Os valores dos coeficientes de determinação do modelo indicam que a variável antecedente prediz adequadamente a alteração das variáveis observadas. Esses coeficientes de regressão estimados além de elevados também são altamente significativos. Todas as variâncias estimadas, tanto aquelas associadas aos erros de medida quanto aquela referente à variável latente, possuem significância estatística.

Apesar da melhora dos índices, após os ajustes realizados, a matriz de resíduos padronizados indica que ainda existem valores altos. Esse tipo de resultado indica que o modelo ainda necessita de ajustes para melhor explicar os dados.

$\mathrm{Na}$ tabela 4, encontram-se os resultados das relações testadas entre as variáveis do modelo. Nesses itens são apresentados os coeficientes de regressão estimados para todos os componentes do modelo, bem como as variâncias dos erros e da variável latente.

Tabela 4

Coeficientes de regressão estimados

\begin{tabular}{lcc}
\hline \hline \multicolumn{1}{c}{ Índice } & $\beta$ & $\mathrm{P}$ \\
\hline \hline Atitudes de aceitação <--- Práticas & 0,68 & 0,00 \\
Atitudes de temor <--- Práticas & 0,04 & 0,50 \\
Atitudes de ceticismo <--- Práticas & $-0,19$ & 0,00 \\
Resultados percebidos <--- Atitudes de aceitação & 0,44 & 0,00 \\
Resultados percebidos <--- Atitudes de temor & 0,29 & 0,00 \\
Resultados percebidos <--- Atitudes de ceticismo & 0,17 & 0,12 \\
\hline \hline
\end{tabular}

A análise dos resultados das relações testadas no modelo permite avaliar se as hipóteses da pesquisa foram corroboradas ou não. 


\section{Discussão}

As informações apresentadas no modelo ajustado pela equação estrutural são suficientes para confirmar parcialmente ou totalmente as hipóteses expostas neste estudo, mesmo que os resultados mostrem que o modelo ainda precisa de ajustes.

A primeira hipótese elaborada para a pesquisa afirma que as práticas de gestão da mudança afetam os resultados percebidos com a mudança. Esta hipótese é confirmada por meio das atitudes frente a mudança, que serve como mediadora do processo, impactando os resultados percebidos, confirmando o que foi dito por Davis e Fisher (2002) e Hayes (2007). Há uma relação positiva e significativa $(\beta=, 34 ; p=, 00)$ entre as práticas de gestão e os resultados percebidos com a mudança organizacional. A hipótese 2 do estudo também foi corroborada pelos resultados na medida em que existe uma relação positiva e significativa $(\beta=, 68 ; p=, 00)$ entre as práticas de gestão da mudança e as atitudes de aceitação das mudanças organizacionais. De acordo com os autores Maurer (2001) e Rao (2015), esta ação pode impactar diretamente na produtividade do indivíduo.

A identificação de uma relação negativa significativa entre as práticas de gestão da mudança e as atitudes de ceticismo em relação a mudança permitem corroborar parcialmente a hipótese 3 do presente estudo. Contudo, as práticas de gestão da mudança parecem não afetar as atitudes de temor frente a mudança organizacional $(\beta=, 04 ; p=, 50)$, o que leva a entender que diante do processo de mudança organizacional, quando há utilização das práticas de gestão de mudança as pessoas se dispõe mais a aceitar o processo que se opor, possibilitando um trabalho mais efetivo por parte de quem conduz a mudança.

As hipóteses 4 e 5 abordam o fato das atitudes em relação a mudança afetarem os resultados percebidos em relação a mudança de maneira positiva ou negativa. De acordo com os resultados apresentados, as atitudes de aceitação afetam positivamente os resultados $(\beta=, 44 ; p=, 00)$ com a mudança organizacional, e portanto, mediam a relação entre práticas de 
gestão da mudança e resultados percebidos com a mudança. Assim sendo, a hipótese 4 foi corroborada, confirmando o pensamento de Damanpour (1991) e Neiva e Paz (2012) mencionado anteriormente. A hipótese 5 do estudo não foi corroborada, porque não há uma relação negativa entre as atitudes de ceticismo e os resultados percebidos com a mudança $(\beta=, 17 ; p=, 12)$. Apesar de existir uma relação positiva entre as atitudes de temor e os resultados percebidos com a mudança $(\beta=, 29 ; \mathrm{p}=, 00)$, não há uma relação entre as práticas de gestão da mudança e as atitudes de temor frente a mudança, como foi dito antes. Portanto, não existe uma relação de mediação quando se considera essas três variáveis. Um resultado surpreendente diz respeito ao fato das atitudes de temor afetarem positivamente os resultados percebidos com a mudança. O que leva a entender que mesmo com medo do que possa acontecer, as pessoas acabam por cooperar com o processo de mudança.

Como limitações do estudo, além do ajuste estatístico para redução dos resíduos apresentados pelo modelo, ressalta-se restrições em relação a amostra e aos instrumentos de atitude frente a mudança e práticas de gestão de mudança.

Limitações devido à amostra vêm da heterogeneidade dos participantes e dos contextos vividos por eles. A presente pesquisa não foi aplicada em uma única organização, ou em tipo particular de negócio, nem tão pouco em um contexto de mudança específico. É possível que a autocorrelação tenha sido minimizada devido à falta de controle no processo de mudança gerada pela heterogeneidade da amostra. Outra característica da amostra é que a maioria dos participantes não possui cargo de liderança. Seria interessante analisar um número maior de gestores, em pesquisas futuras, para verificar os resultados percebidos e as atitudes frente a mudanças por meio das práticas de gestão de mudança. De acordo com o resultado apresentado na presente pesquisa, os respondentes, sejam eles líderes ou liderados, enxergam as práticas de gestão de mudança e seus impactos da mesma maneira. 
Quanto às limitações relativas ao instrumento de atitudes frente a mudança, pode-se dizer que, mesmo o instrumento tendo evidências de validade, os dados demostram que possa existir uma variável não considerada, ainda desconhecida, que influencie nos resultados, já que erros foram apresentados no modelo. Sugere-se que as estruturas fatoriais do instrumento sejam revisadas, bem como a realização de nova pesquisa em um contexto claro e específico. Quanto ao instrumento de práticas de gestão de mudança, a limitação pode estar relacionada ao processo de tradução transcultural do questionário. Ainda que os devidos cuidados quanto a tradução e interpretação dos itens tenham sido tomados, não houve a preocupação para saber se os significados de alguns termos utilizados no questionário aplicado no Brasil são iguais aos utilizados no Chile, onde foi aplicada a pesquisa original. Recomenda-se uma revisão transcultural no instrumento de práticas de gestão da mudança em estudos futuros.

Uma vez que os contextos econômico, social e tecnológico estão em constantes mudanças, estudos que realizem avaliações da mudança organizacional são de grande relevância. Do ponto de vista prático, pode-se entender que quanto maior for o desempenho individual esperado pelas empresas, maior a ênfase que deve ser dada às práticas de gestão de mudança, pois verificou-se impacto na aceitação e efeitos percebidos. Espera-se que os resultados contribuam para construir planos de ação que tornem os processos de mudança mais naturais e satisfatórios dentro das organizações, principalmente no modo que são subsidiados pela utilização de práticas de gestão de mudança.

\section{Referências}

Afande, F. O. (2015). Factors that Influence Organizational Transformation: A Case of Life Insurance Companies in Kenya. Journal of Marketing and Consumer Research, 8, 111-142. 
Ambos, T. C., \& Birkinshaw, J. (2010). How do new ventures evolve? An inductive study of archetype changes in science-based ventures.Organization Science, 21(6), 1125-1140.

Anderson , D.,\& Anderson, L. S. (2001). Beyond change management: advanced strategic for today's transformational leaders. San Francisco: Jossey-Bass.

Armenakis, A.A , Brown, S \& Mehta, A. (2011) Organizational Culture: Assessment and Transformation. Journal of Change Management, 11(3), 305-328.

Balogun, J. (2006). Managing change: Steering a course between intended strategies and unanticipated outcomes. Long Range Planning, 39(1), 29-49. DOI: 10.1016/j.lrp.2005.02.010

Barends, E., Janssen, B., ten Have, W., \& ten Have, S. (2014). Difficult but doable: Increasing the internal validity of organizational change management studies. Journal of Applied Behavioral Science, 50, 50-54.

Barron, E., Riby, L. M., Greer, J., \& Smallwood, J. (2011). Absorbed in thought the effect of mind wandering on the processing of relevant and irrelevant events. Psychological science. DOI: 10.1177/0956797611404083

Battilana, J., Gilmartin, M., Sengul, M., Pache, A. C., \& Alexander, J. A. (2010). Leadership competencies for implementing planned organizational change. The Leadership Quarterly, 21(3), 422-438.

Birkinshaw, J. (2010). The critical need to reinvente management. British Journal of Management, 21(1) 4-11. DOI: 10.1111/j.1467-8616.2010.00636.x

Bortolotti, S. L., Souza Junior, A. F., \& Andrade, D. F. (2009). Uma proposta de modelo para avaliar a resistência à mudança organizacional. Anais do VI Simpósio de Excelência em Gestão e Tecnologia (SEGET), 1-12.

Burke, W. W., \& Litwin, G. H. (1992). A causal model of organizational performance and change. Journal of Management,18, 523-545. 
Burke, W.W. (2011). A perspective on the field of organization development and change: The Zeigarnik effect.Journal of Applied Behavioral Science, 47, 143-167.

Burnes, B. (2004). Kurt Lewin and the Planned Approach to Change: A Re-appraisal. Journal of Management Studies, 41(6), 977-1002.

Burtonshaw-Gunn, S. A. \& Salameh, M. G. (2010) Change management contribution to organisational performance. Human Capital Review. 2(12), 1-6.

Cameron E., \& Green. M, (2012). Making Sense of Change Management: A Complete Guide.

Castel, P., Friedberg, E., (2009) Institutional Change as an Interactive Process: The Case of the Modernization of the French Cancer Centers Organization. Science, 21(2), 311 330.

Cinite, I., Duxbury, L.E., Higgins, C. (2009). Measurement of perceived organizational readiness for change in the public sector. British Journal of Management, 20(2), 265277. DOI: $10.1111 / \mathrm{j} .1467-8551.2008 .00582 . \mathrm{x}$

Currie, G.,Lockett, A, Suhomlinova, O. (2009)' Leadership and institutional change in the public sector: The case of secondary schools in England. The Leadership Quarterly, 20(5), 664-679. doi:10.1016/j.leaqua.2009.06.001

Dahl, M.S. (2011). Organizational Change and Employee Stress. Management Science, 57 (2), $240-256$.

Davis, D., \& Fisher, T. (2002). Attitudes of middle managers to quality-based organisational change. Managing Service Quality: An International Journal, 12(6), 405-413. 
Domingos, S. G., \& Neiva, E. R. (2014). Percepção dos Funcionários sobre Mudanças Transacionais e Transformacionais em uma Organização Pública. $R A C, 18(2), 118$ 138.

Ford, J. D., Ford, L. W., \& D'Amelio, A. (2008). Resistance to change: The rest of the story. Academy of Management Review, 33, 362-377. DOI: 10.5465/AMR.2008.31193235.

Gilley, A, Gilley, J.W \& McMillan. (2009). Organizational Change: Motivation, Communication, and Leadership Effectiveness. Performance Improvement Quarterly, 21(4), 75-94.

Grant, D., Marshak R.J. (2011). Toward a Discourse-Centered Understanding of Organizational Change. Journal of Applied Behavioral Science. doi:10.1177/0021886310397612

Hardy, C., Thomas, R.(2011). Reframing resistance to organizational change. Scandinavian Journal of Management, 27(3), 322-331.

Hayes, J. (2002). The theory and practice of change management. New York: Palgrave.

Hayes, J. (2007). Evaluating a leadership development program. Organization Development Journal, 25(4), P89.

Jansson , N. (2013). Organizational change as practice: a critical analysis. Journal of Organizational Change Management, 26(6), 1003-1019.

Jarzabkowski, P., \& Seidl, D. (2008). The role of meetings in the social practice of strategy. Organization Studies, 29(11), 1391-1426.

Jian, G. (2011). Articulating circumstance, identity and practice: toward a discursive framework of organizational changing. Organization, 18(1), 45-64. 
Kline, R. B. (2011). Principles and practice of structural equation modeling. New York: Guiford Press.

Kotter, J. P. (2000). Liderando mudança. 3 Ed. Rio de Janeiro: Campus.

Levay, C. (2010). Charismatic leadership in resistance to change. The Leadership Quarterly, 21(1), 127-143.

Lewis, L. K., Schmisseur, A. M., Stephens, K. K., \& Weir, K. E. (2006). Advice on Communicating During Organizational Change The Content of Popular Press Books. Journal of business Communication, 43(2), 113-137.

Lines, R. (2005). The Structure and Function of Attitudes Toward Organizational Change. Human Resource Development Review, 4(1), 8-32. London.3rd Ed.

Lüscher, L. S., \& Lewis, M. W. (2008). Organizational change and managerial sensemaking: Working through paradox. Academy of Management Journal, 51(2), 221-240.

Maurer, T. J. (2001). Career-relevant learning and development, worker age, and beliefs about self-efficacy for development..Journal of Management. 2(27), 123-140.

McDonald, R. P., \& Ho, M.-H. R. (2002). Principles and practice in reporting structural equation analyses. Psychological Methods, 7(1), 64-82.

Nastase, M., Giuclea, M., Bold, O. (2012): The Impact of Change Management in Organizations - A Survey of Methods and Techniques for a Successful Change, Review of International Comparative Management. 13, 5-16.

Neiva, E. R., \& Pantoja, M. J. (2011). Aprendizagem e Mudança Organizacional: Das Relações Entre Atitudes Frente à Mudança e Estratégias de Aprendizagem no Trabalho. Revista Interamericana de Psicologia, 45 (2), 145-156. 
Neiva, E. R., \& Paz, M. T. (2012). Percepção de mudança individual e organizacional: o papel das atitudes, dos valores, do poder e da capacidade organizacional. Revista de Administração, 47 (1), 22-37.

Neiva, E. R., Ros, M., \& Paz, M. T. (2004). Validacion de una escala de actitudes ante el cambio organizacional. Revista de Psicologia del Trabajo e das Organizaciones, 20(1), 9-30.

Oreg, S., Vakola, M., Armenakis, A. (2011). Change Recipients' Reactions to Organizational Change A 60-Year Review of Quantitative Studies. Journal of Applied Behavioral Science, 47(4), 461-524.

Pache, A. C., \& Santos, F. (2010). When worlds collide: The internal dynamics of organizational responses to conflicting institutional demands. Academy of Management Review, 35(3), 455-476.

Pache, A., Santos, F. (2010). When Worlds Collide: The Internal Dynamics of Organizational Responses to Conflicting Institutional Demands. Academic Management Review, $35(3), 455-476$.

Pasquali, L. (2012). Analise Fatorial para pesquisadores. Brasília: LabPAM.

Picchi, T. d. (2010). Educação Corporativa: ajuste com sistemas de gestão de pessoas e resultados de treinamento nos níveis individual e organizacional. Dissertação de Mestrado. Brasília, DF: Universidade de Brasília.

Ranieri, A. B. (2011). Change management practices: Impact on perceived change results. Journal of Business Research, 64, 266-272. 
Rao, M.S. (2015) "The tools and techniques of effective change management: Why some reformers succeed while others fail". Human Resource Management International Digest, 23(1), 35-37.

Rouleau, L., \& Balogun, J. (2011). Middle managers, strategic sensemaking, and discursive competence. Journal of Management Studies, 48(5), 953-983.

Sande, O. A., Walela, K. B., \& Wamukoya, O. (2015). Change Management And Performance Of Public Secondary Schools In Siaya Sub County. International journal of scientific \& technology, 4(4), 162-174.

Sande, O.A., Walela,K.B. Wamukoya, O. (2015). Change Management And Performance Of Public Secondary Schools In Siaya Sub County International Journal of Scientific \& Technology Research. 4 (4), 163-174.

Schafer, J. A. (2010). Effective leaders and leadership in policing: traits, assessment, development, and expansion. Policing: An International Journal of Police Strategies \& Management, 33(4), 644-663.

Schatzki, T.R., Cetina, K.K, Savigny, E.(2001) The Practice Turn in Contemporary Theory. Routledge, London.

Schreyögg, G., \& Sydow, J. (2011). Organizational path dependence: A process view. Organization Studies, 32(3), 321-335.

Self, D., Armenakis, A, \& Scharaeder, M. (2007). Organisaconal change, contente, process and contexto. A simultaneous analysis of employee reactions. Journal of Change Management, 7(2), 211-219.

Sellmann, M. Z., \& Marcondes, R. C. (2010). A gestão da mudança em ambientes dinâmicos: um estudo no setor das telecomunicações. Cadernos ebape.br, 8(4), 753-781.

Senior, B. (2002). Organisational Change,, 2nd ed, London: Prentice Hall.

Sims, S. (2002). Changing the way we manage change. Westport: Quorum Books. 
Smith, W.K., \& Lewis, M.W. (2011): "Toward a theory of paradox: a dynamic equilibrium model of organizing. Academy of Management Review, 36 (2), 381-403.

Sonenshein, S.(2010). We're Changing - Or Are We? Untangling the Role of Progressive, Regressive, and Stability Narratives During Strategic Change Implementation. Academy of Management Journal, 53(3), 477-512. doi:10.5465/AMJ.2010.51467638.

Stensaker, I. G., \& Langley, A. (2010). Change management choices and trajectories in a multidivisional firm. British Journal of Management, 21(1), 7-27.

Stoner, J.A.F, Freeman, R.E. \& Junior, D.R. (2005). Subjective indicators as a gauge for improving organizational well-being. An attempt to apply the cognitive activation theory to organizations. Prentice Hall, 2009 - 6th ed.

Todd, A. (1999). Managing radical change. Long Range Planning, 32(2), 237-244.

Van de Ven, A. H., \& Poole, M. S. (2005). Alternative approaches for studying organizational change. Organization studies, 26(9), 1377-1404.

Van de Ven, A. H., \& Sun, K. (2011). Breakdowns in implementing models of organization change. The Academy of Management Perspectives, 25(3), 58-74. DOI: 10.5465/AMP.2011.63886530.

Weick, K. E., \& Quinn, R. E. (1999). Organizational change and development. Annual review of psychology, 50(1), 361-386.

Whittle, A., Suhomlinova, O., \& Mueller, F. (2010). Funnel of interests: The discursive translation of organizational change. The Journal of Applied Behavioral Science, 46(1), 16-37.

Woodman, R. W. (2014). The Science of Organizational Change and the Art of Changing Organizations. The Journal of Applied Behavioral Science, 50(4), 463-477. 